\title{
TUNNELING MAGNETORESISTANCE IN FERROMAGNETIC JUNCTIONS: BIAS DEPENDENCE
}

\author{
J. Barnaś* S.F. Lee, P. Holody, L.F. Schelp, F. Petroff and A. Fert \\ Unite Mixte de Physique CNRS/Thomson \\ Domaine de Corbeville, 91404 Orsay, France
}

Electron tunneling between two ferromagnetic electrodes across an insulating barrier is analysed theoretically and experimentally. The barrier is either uniform or it includes a layer of small magnetic metallic particles. Particular attention is paid to the origin of the tunneling magnetoresistance and its bias dependence, as well as to the effects due to Coulomb blockade.

PACS numbers: 73.40.Gk, 73.40.Rw, 75.70.-i

By tunneling magnetoresistance (TMR) we mean in this paper a drop $\Delta R$ in the junction resistance when the magnetizations of two ferromagnetic electrodes rotate from antiparallel to parallel alignment. The effect was observed first by Julliere [1] in $\mathrm{Co} / \mathrm{Ge} / \mathrm{Fe}$ junctions. Similar observations were reported later also in other systems $[2,3]$. Recent experimental data show additionally that TMR significantly decreases with increasing voltage $V$ [4].

Two microscopic approaches have been developed to account for TMR in ferromagnetic junctions. One of them takes into account many-body features of the corresponding electronic wave functions in ferromagnetic electrodes [5]. In the second approach, on the other hand, one-electron wave functions are used to calculate the tunneling current [6]. In both models a complex electronic band structure of a ferromagnet was approximated by the one-band model with free-electron-like spin-polarized parabolic bands. However, there are some additional features of the electronic structure, which can contribute to TMR, and which have not been taken into account in the above models. The most important seems to be the $s-d$ hybridization in the ferromagnetic electrodes [7] as well as at the electrode/barrier interfaces. Apart from this, the barrier height for $d$ electrons can differ remarkably from the barrier height for $s$ electrons [8]. Thus, the tunneling probability for a particular electronic state depends on the rate of $s-d$ hybridization. This, on

*Permanent address: Magnetism Theory Division, Institute of Physics, A. Mickiewicz University, Matejki 48/49, 60-769 Poznań, Poland. 
taking into account the spin asymmetry caused by a spin-split of the $d$-band, can contribute to TMR.

To emphasize the role of $s-d$ hybridization we assume a simple electronic structure (the same for both electrodes) which includes one parabolic band of $s$ electrons and one spin-split band of $d$ electrons. For simplicity, we assume that the $d$ electrons are strongly localized and neglect the effects due to a finite width of the $d$ band. Let the corresponding localized $d$ levels be at the energy $U_{d+}$ for spin-majority electrons and $U_{d-}$ for spin-minority electrons $\left(U_{d+}<U_{d_{-}}\right)$. The $s$ and $d$ electronic states are hybridized inside the electrodes with the corresponding coupling parameter equal to $V^{s d}$ for both spin orientations. Interfacial hybridization will be neglected here. A particular electronic state $|\psi\rangle$ is generally a superposition of the $s$-like $|s\rangle$ and $d$-like $|d\rangle$ components, $|\psi\rangle=u|s\rangle+v|d\rangle$, where the parameters $u$ and $v$ can be found from the diagonalization procedure. We also assume that the transition probability for "pure" $d$ states is significantly smaller (due to a larger barrier height) than the corresponding transition probability for "pure" $s$ states.

Taking into account the approximations described above, the tunneling current $j$ per unit area can be calculated in the zero-temperature limit from the formula

$$
j=\frac{e}{4 \pi^{2} \hbar} \sum_{\sigma} \int_{E_{\mathrm{F}}-\mathrm{eV}}^{E_{\mathrm{F}}} \mathrm{d} E \int_{0}^{k_{\| \sigma}^{\max }} u_{\mathrm{L} \sigma}^{2}(E) u_{\mathrm{R} \sigma}^{2}(E+e V) k_{\|} \exp (-\eta) \mathrm{d} k_{\|},
$$

where $e$ is the electron charge $(e>0)$ and $k_{\|}\left(k_{\|}=\left|k_{\|}\right|\right)$is the in-plane component of the electron wave vector, which is assumed to be conserved in the tunneling events in planar junctions. The upper integration limit, $k_{\| \sigma}^{\max }$, is determined by the energy conservation and the electronic structure. $E_{\mathrm{F}}$ is the Fermi energy (measured from the bottom of the $s$ band), while $u_{\mathrm{L} \sigma}(E)$ and $u_{\mathrm{R} \sigma}(E+e V)$ are the diagonalization coefficients introduced above and taken respectively at the energy $E$ in the left (source) electrode and at the energy $E+e V$ in the right (drain) electrode. Finally, in the WKB approximation $\eta$ is given by $\eta=2 \int_{0}^{d_{\mathrm{b}}} k_{x} \mathrm{~d} x$, where $k_{x}=\left[\left(2 m / \hbar^{2}\right)\left(E_{\mathrm{F}}+U_{\mathrm{b}}(x)-E\right)+k_{i}^{2}\right]^{1 / 2}$. Here, $U_{\mathrm{b}}(x)$ describes profile of the effective barrier potential (which includes the effect of applied voltage) and $d_{0}$ is the barrier thickness ( $x=0$ and $x=d_{\mathrm{b}}$ are the classical turning points).

Figure 1 shows TMR (defined as $\Delta R / R_{\mathrm{p}}$, where $R_{\mathrm{p}}$ is the total junction resistance in the parallel configuration) as a function of the voltage $V$. Different curves correspond there to different positions of the $d$ level for spin-minority electrons. The other parameters are the same for all curves. The only spin asymmetry is then due to spin dependent position of the $d$ levels and the zero-bias TMR is crucially dependent on this asymmetry. For all curves TMR decreases with increasing voltage and the higher zero-bias TMR, the larger rate of this decrease.

Consider now the situation when the magnetizations of both electrodes are parallel and the barrier includes a layer of ferromagnetic metallic grains with their magnetic moments either parallel or antiparallel to the electrodes magnetization. When the typical capacitance $C$ of a single grain is small, so that the charging energy $e^{2} /(2 C)$ is comparable with the thermal energy $k T$, the effects due to Coulomb blockade become important [9]. 


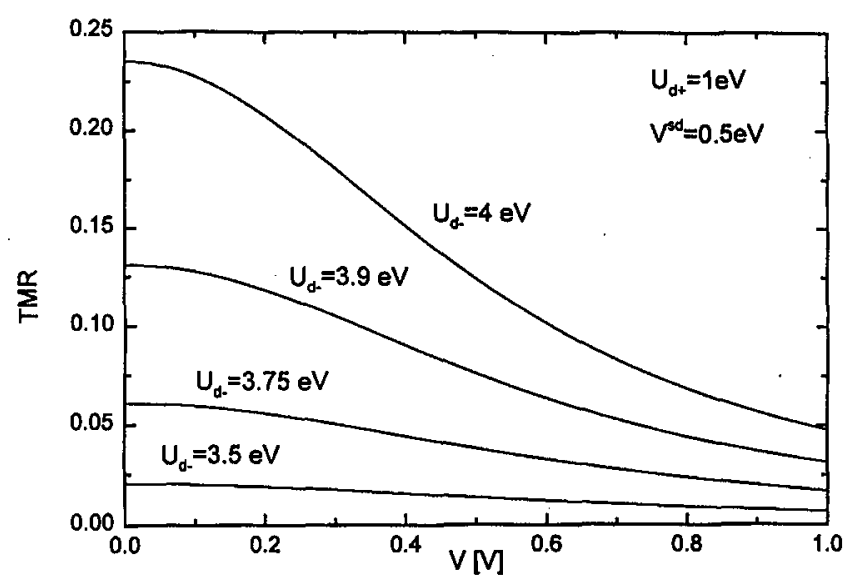

Fig. 1. TMR in a simple planar junction as a function of the voltage, calculated for $E_{\mathrm{F}}=4.5 \mathrm{eV}, d_{\mathrm{b}}=20 \AA, U_{\mathrm{b}}(x)=\mathrm{const}=1.2 \mathrm{eV}$ (for $V=0$ ) and for indicated values of $U_{d-}, U_{d+}$ and $V^{s d}$.

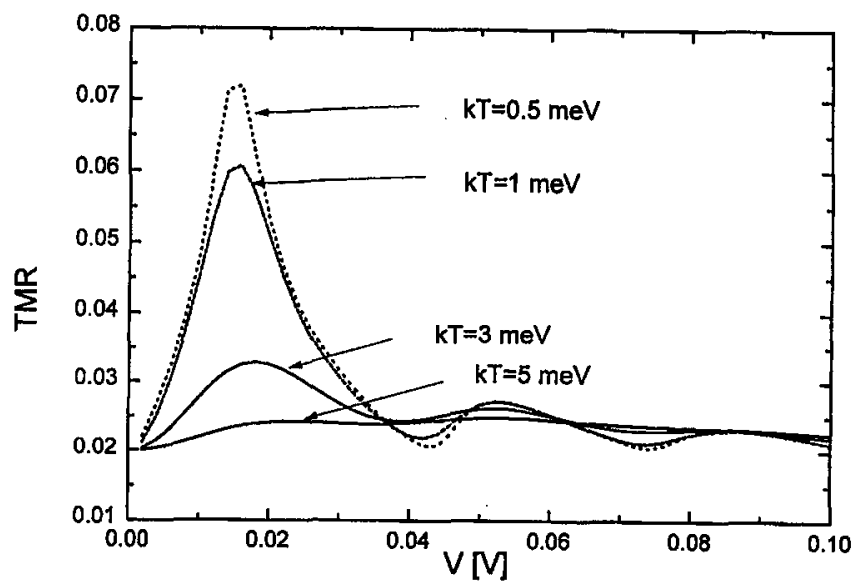

Fig. 2. TMR in a double microjunction for $R^{\mathrm{P}}{ }_{1+}=R_{1-}^{\mathrm{p}}=150 \mathrm{M} \Omega, R_{2+}^{\mathrm{p}}=70 \mathrm{M} \Omega$, $R_{2-}^{\mathrm{p}}=30 \mathrm{M} \Omega, C_{1} / C_{2}=2, E_{\mathrm{c}}=10 \mathrm{meV}, R^{\mathrm{a}} \mathrm{p}_{\alpha+}=R_{\alpha-}^{\mathrm{ap}}=\left(R_{\alpha_{+}}^{\mathrm{p}} R_{\alpha-}^{\mathrm{p}}\right)^{1 / 2}($ for $\alpha=1$ and $\alpha=2$ ) and for indicated values of the thermal energy.

Since the whole junction can be considered as a series of single-grain junctions connected in parallel, let us consider first a double ferromagnetic junction consisting of two electrodes (leads) and a grain in between. If the junction capacitances are $C_{1}$ and $C_{2}$, then $C=C_{1}+C_{2}$. We assume that the energy and spin relaxation times on the grain are shorter than the time between two successive tunneling events. Consequently, the electrons which tunnel into the grain relax to the Fermi level before the next tunneling event, and this Fermi level is common 
for both spin orientations. Since the tunneling probabilities depend on the number of excess electrons on the grain, the problem is generally a stochastic one and the current can be found by solving the appropriate master equation [10].

Since the typical scale for the bias dependence of TMR shown in Fig. 1 as well as for the nonlinear terms in the $I-V$ characteristics is of the order of $10^{2} \mathrm{mV}$, while for $E_{\mathrm{c}} \approx 10 \mathrm{meV}$ the typical scale for the Coulomb blockade effects is of the order of $10 \mathrm{mV}$, we assume constant junction resistances $R_{1 \sigma}^{\mathrm{p}}$ and $R_{2 \sigma}^{\mathrm{p}}\left(R_{1 \sigma}^{\mathrm{ap}}\right.$ and $R_{2 \sigma}^{\mathrm{ap}}$ ), respectively for the right and left junctions in the parallel (antiparallel) configuration.

Figure 2 shows TMR for several values of the thermal energy $k T$. The Coulomb blockade effects lead to oscillations in TMR with increasing $V$. The oscillation period is determined by the charging energy and the oscillation amplitude decreases with increasing $V$. Amplitude of those oscillations also decreases with increasing temperature and vanishes when the thermal energy is of the order of the charging energy.

Figure 3 presents experimental results obtained on a macroscopic $\mathrm{Co} /$ $\mathrm{Al}_{2} \mathrm{O}_{3}(\mathrm{Co}) / \mathrm{Co}$ junction, which consists of two Co electrodes and $\mathrm{Al}_{2} \mathrm{O}_{3}$ barrier with a layer of Co clusters inside the barrier. Note that TMR is defined there in a slightly different way than in Figs. 1 and 2. The results clearly indicate the

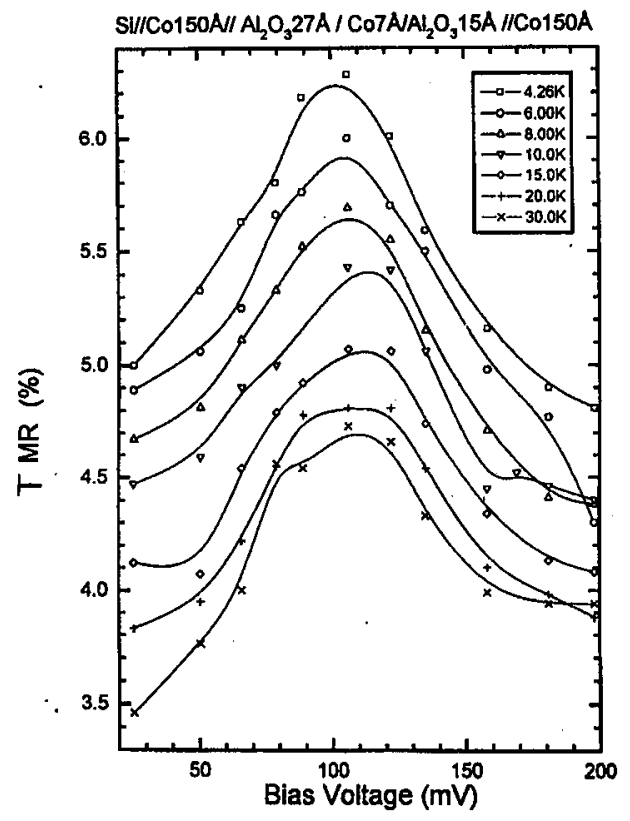

Fig. 3. TMR as a function of the bias voltage obtained on a macroscopic $\mathrm{Co} /$ $\mathrm{Al}_{2} \mathrm{O}_{3}(\mathrm{Co}) / \mathrm{Co}$ junction with a layer of Co grains inside the barrier. The diameter of the spherical clusters is about $4 \mathrm{~nm}$. TMR is defined here as $\left.\left(\Delta(\mathrm{d} V / \mathrm{d} I) /(\mathrm{d} V / \mathrm{d} I)_{\mathrm{p}}\right) \times 10^{2}\right)$, where $\Delta(\mathrm{d} V / \mathrm{d} I)$ is a difference in the derivative $(\mathrm{d} V / \mathrm{d} I)$ between the antiparallel and parallel configurations. 
presence of two contributions to the bias dependence of TMR, as discussed above. They also show that TMR decreases with increasing temperature.

One of us (JB) acknowledges hospitality during his stay at the Unite Mixte de Physique CNRS/Thomson, as well as support through the Research Project 2 P03B 16510 of the Committee for Scientific Research (Poland). This work was supported in part by the EEC through Esprit project No 20-027 $\left(\mathrm{NM}^{2}\right)$ and Brite project BE96-3407 (Hot SEAMS).

\section{References}

[1] M. Julliere, Phys. Lett. A 54, 225 (1975).

[2] T. Miyazaki, T. Yaoi, S. Ishio, J. Magn. Magn. Mater 98, L7 (1991).

[3] J. Nowak, J. Raułuszkiewicz, J. Magn. Magn. Mater 109, 79 (1992).

[4] J.S. Moodera, L.R. Kinder, T.M. Wong, R. Meservey, Phys. Rev. Lett 74, 3273 (1995).

[5] M.B. Stearns, J. Magn. Magn. Mater. 5, 167 (1977).

[6] J.C. Slonczewski, Phys. Rev. B 39, 6995 (1989).

[7] J.N. Chazalviel, Y. Yafet, Phys. Rev. B 15, 1062 (1977).

[8] S.F. Alvarado, Phys. Rev. Lett 75, 513 (1995).

[9] For a review see Single Charge Tunneling, Eds. H. Grabert, M.H. Devoret, NATO ASI Series, Vol. 294, Plenum Press, New York 1992.

[10] M. Amman, R. Wilkins, E. Ben-Jacob, P.D. Maker, R.C. Jaklewic, Phys. Rev. B 43, 1146 (1991). 